\title{
Determination of low-strain interfaces via geometric matching
}

Jelver, Line; Larsen, Peter Mahler; Stradi, Daniele; Stokbro, Kurt; Jacobsen, Karsten Wedel

Published in:

Physical Review B

Link to article, DOI:

10.1103/PhysRevB.96.085306

Publication date:

2017

Document Version

Publisher's PDF, also known as Version of record

Link back to DTU Orbit

Citation (APA):

Jelver, L., Larsen, P. M., Stradi, D., Stokbro, K., \& Jacobsen, K. W. (2017). Determination of low-strain interfaces via geometric matching. Physical Review B, 96(8), [085306 ]. https://doi.org/10.1103/PhysRevB.96.085306

\section{General rights}

Copyright and moral rights for the publications made accessible in the public portal are retained by the authors and/or other copyright owners and it is a condition of accessing publications that users recognise and abide by the legal requirements associated with these rights.

- Users may download and print one copy of any publication from the public portal for the purpose of private study or research.

- You may not further distribute the material or use it for any profit-making activity or commercial gain

- You may freely distribute the URL identifying the publication in the public portal

If you believe that this document breaches copyright please contact us providing details, and we will remove access to the work immediately and investigate your claim. 


\title{
Determination of low-strain interfaces via geometric matching
}

\author{
Line Jelver, ${ }^{1,2}$ Peter Mahler Larsen, ${ }^{2}$ Daniele Stradi, ${ }^{1}$ Kurt Stokbro, ${ }^{1}$ and Karsten Wedel Jacobsen ${ }^{2, *}$ \\ ${ }^{1}$ QuantumWise A/S, Fruebjergvej 3, DK-2100 Copenhagen, Denmark \\ ${ }^{2}$ Department of Physics, Technical University of Denmark, DK-2800 Kongens Lyngby, Denmark
}

(Received 10 May 2017; published 22 August 2017)

\begin{abstract}
We present a general method for combining two crystals into an interface. The method finds all possible interfaces between the crystals with small coincidence cells and identifies the strain and area of the corresponding two-dimensional cells of the two crystal surfaces. We apply the method to the two semiconductor alloys $\operatorname{InAs}_{1-x} \mathrm{Sb}_{x}$ and $\mathrm{Ga}_{x} \mathrm{In}_{1-x}$ As combined with a selection of pure metals or with NbTiN to create semiconductor/superconductor interfaces. The lattice constant of the alloy can be tuned by composition and we can extract the alloy lattice parameters corresponding to zero strain in both the metal and the alloy. The results can be used to suggest new epitaxially matched interfaces between two materials.
\end{abstract}

DOI: 10.1103/PhysRevB.96.085306

\section{INTRODUCTION}

The development of modern technology has become increasingly dependent on knowledge of interfaces at the atomic scale. As the size of electronic devices decreases, interfaces become an increasingly dominant part of the system and thus become the limiting factor for device performance [1-3]. Many difficulties are thus related to obtaining a stable and defect-free interface. When the materials are not commensurate, a large strain can build up at the interface, and result in defects and unstable interface geometries. To this end, it can be beneficial to use an alloy as one of the interface materials. By changing the alloy composition, the lattice constant for one of the interface materials can be tuned, thereby obtaining commensurate lattices across the interface. Many future devices can therefore be expected to be designed from alloys. A growing field, where alloys are commonly used, is the fabrication of core-shell nanowires. These nanowires have numerous applications, e.g., for photodetectors [4], photoelectrodes [5], and thermoelectric devices [6].

The prediction of the stability of an interface is difficult [7] and the commonly used approach is trial and error where many samples must be grown before it can be concluded whether a stable interface can be formed or not. In this paper, we present a crystal matching method which permits the combination of any two crystals and provides information on the crystal surfaces, if any, that allow for a low-strain epitaxial interface. The method is solely based on geometrical considerations of the possible surface cells of the two crystals and it leads to an identification of interfaces where both the strain and the size of the coincidence interface cell are small. Having low stress and a small interface cell does not by itself guarantee a stable interface, as the atomic structure of the interface may also play an important role. However, the simple geometrical criteria provide a good starting point for further experimental or theoretical investigations.

In a previous article [8], some of the authors have shown how to find good matches between specific surfaces of two crystals and a related, but more simplistic, method for such a two-dimensional (2D) match has also previously been

*kwj@fysik.dtu.dk proposed [9]. With the present method, all possible crystal orientations and surfaces are investigated at the same time. Furthermore, we introduce a scaling parameter between the two crystals which can be tuned to gradually change the size of one of the crystal structures while keeping the other fixed. The scaling parameter mimics the situation where the lattice constant of one of the materials can be tuned by modifying the alloy composition, and it provides a convenient parameter for analyzing and understanding the interface-matching problem. We derive an analytical relation between the scaling parameter and the minimal strain, and demonstrate its usefulness in Sec. IV, where we investigate the matches between an arbitrary fcc crystal and an arbitrary bec crystal.

The introduction of the scaling parameter makes the method an ideal tool for investigating interfaces containing alloys. In Sec. V, we apply the method to the two semiconductor alloys $\mathrm{InAs}_{1-x} \mathrm{Sb}_{x}$ and $\mathrm{Ga}_{x} \mathrm{In}_{1-x}$ As combined with a range of metals ( $\mathrm{Al}, \mathrm{Ni}, \mathrm{Cu}, \mathrm{Ag}, \mathrm{Au}, \mathrm{Pb}, \mathrm{V}, \mathrm{Fe}, \mathrm{Nb}$, and $\mathrm{Co}$ ) and for each metal we predict the alloy lattice constants which lead to strain free interfaces. InAs and InSb are, for example, used in semiconductor-superconductor core-shell nanowires for the realization of zero-energy localized Majorana modes [10-15]. The results obtained here therefore provide some guidelines for promising new combinations of semiconductor alloy compositions and metals for core-shell nanowires. Promising results for the generation of Majorana modes have also been shown for an InSb-Nb ${ }_{1-x} \mathrm{Ti}_{x} \mathrm{~N}$ interface [16]. The final part of this work is to investigate how the two semiconductor alloys match with this superconducting alloy.

\section{INTERFACE ENERGETICS}

The method we are going to describe below makes it possible to identify interfaces with small coincidence cells where only a small strain is required. The model thus takes only the material lattices into account, but not the atomic details. To what extent such interfaces will be stable is highly dependent on the particular materials and the character of the bonding. To discuss this we consider the situation where two materials A and $\mathrm{B}$ are homogeneously strained and put together to form an interface. The energy difference $\Delta E$ associated with straining the materials and forming the interface can be divided into 
three contributions,

$$
\Delta E=\Delta E^{\text {int }}+\Delta E^{\text {surf. strain }}+\Delta E^{\text {bulk strain }},
$$

where the interface term is the energy gained by forming the interface from the surfaces at fixed strain,

$$
\Delta E^{\mathrm{int}}=E_{\mathrm{AB}}^{\mathrm{int}}\left(\epsilon_{A}, \epsilon_{B}\right)-E_{A}^{\mathrm{surf}}\left(\epsilon_{A}\right)-E_{B}^{\mathrm{surf}}\left(\epsilon_{B}\right) .
$$

The second term is the energy change in the surface energy because of the strain,

$$
\begin{aligned}
\Delta E^{\text {surf. strain }}= & E_{A}^{\text {surf }}\left(\epsilon_{A}\right)-E_{A}^{\text {surf }}\left(\epsilon_{A}=0\right) \\
& +E_{B}^{\text {surf }}\left(\epsilon_{B}\right)-E_{B}^{\text {surf }}\left(\epsilon_{B}=0\right) .
\end{aligned}
$$

This contribution can be both positive or negative and we shall in the following assume that this term can be considered small. The third term is the energy cost associated with straining the bulk of the materials,

$$
\begin{aligned}
\Delta E^{\text {bulk strain }}= & E_{A}^{\text {bulk }}\left(\epsilon_{A}\right)-E_{A}^{\text {bulk }}\left(\epsilon_{A}=0\right) \\
& +E_{B}^{\text {bulk }}\left(\epsilon_{B}\right)-E_{B}^{\text {bulk }}\left(\epsilon_{B}=0\right) .
\end{aligned}
$$

If we assume that the dependency of the surface energy with strain can be neglected, we expect to get a stable interface if the energy gain from the interface $\Delta E^{\text {int }}$ dominates the cost from straining the bulk of the materials $\Delta E^{\text {bulk strain }}$. The model suggested here identifies interface matches with low-strain and small interface coincidence cells. The small strain will clearly tend to minimize the bulk strain energy as this scales quadratically with the strain. A more questionable assumption is that a small interface cell will lead to strong bonding at the interface. The bonding will often depend quite sensitively on the atomic structure at the interface, which is not considered at all by the model. However, in many cases where the interface cell is small, a translation of one of the surfaces relative to the other makes it possible to obtain favorable bonding configurations which are then repeated over the entire interface, leading to general high stability. However, if the interface cell is large, the atomic bonding configurations will often vary considerably over the cell, so that in some parts of the cell favorable bonding configurations are obtained but in other parts not. Overall this leads to weaker bonding [17].

Another advantage of a small coincidence cell is that it may be more stable with respect to shear in the interface. For a small cell, a large corrugation in the energy landscape as a function of displacement of one of the materials relative to the other one along the interface can be expected. The bonds across the interface will respond to the shear "in synchrony" leading to large variation. For a larger cell where some bonds are strong and others are weak, the bonds will respond differently to the shear presumably leading to a smaller energy corrugation.

If the materials are thick, the strain has to be very small since the bulk strain energy grows in proportion to the thickness. In this limit, only interface matches at very low strain will be acceptable. However, in practice this limit may also lead to incommensurate interfaces or defects at or close to the interface, situations clearly beyond what a simple lattice model can account for. Similarly, if the interface bonding energy is very small and has a small corrugation, as is, for example, the case with van der Waals bonding, stable interfaces with large moiré-pattern coincidence cells or even incommensurate cells may occur. This situation can arise even for very thin films as, for example, in the case of graphene on some metal surfaces, where the interaction is weak. Despite the fact that a graphene layer is atomically thin, the strong interatomic bonding within the graphene layer results, for some metals, in little accommodation of the surface and large coincidence cells as a result $[18,19]$.

\section{CRYSTAL MATCHING METHOD}

Our method for creating an interface between two crystals is general and based on 2D cells of the two crystal surfaces. The 3D vectors of the crystal, defining these surface cells, are projected from $\mathbb{R}^{3}$ to $\mathbb{R}^{2}$ as illustrated in Fig. 1(a). We begin by explaining the method behind matching two such cells and extracting the related strain.

Let the surface cell of the first crystal be defined by two vectors $\mathbf{u}_{1}$ and $\mathbf{u}_{2}$, where $\mathbf{u}_{1}=\left[u_{1 x}, u_{1 y}\right]^{T}$, as shown in Fig. 1(a). Similarly, let $\mathbf{v}_{1}$ and $\mathbf{v}_{2}$ denote the two vectors which define the surface cell of the second crystal. Then, the affine transformation $\mathbf{A}$ which maps $\left[\mathbf{u}_{1}, \mathbf{u}_{2}\right]$ onto $\left[\mathbf{v}_{1}, \mathbf{v}_{2}\right]$ is given by

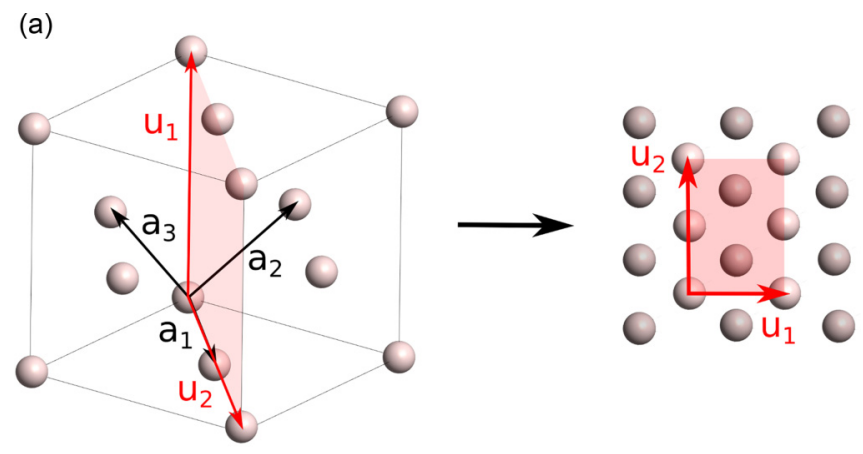

(b)
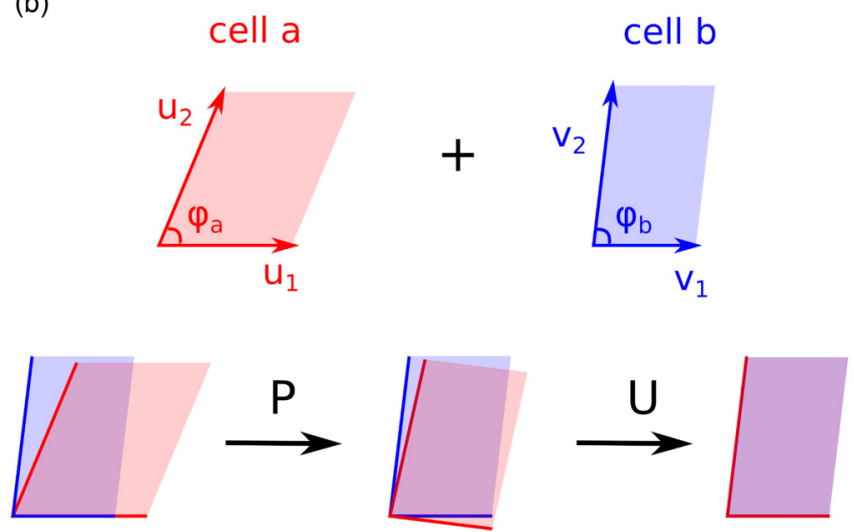

FIG. 1. The method for finding and matching the 2D surface cells of two crystals. (a) The lattice vectors of the first crystal $\left(\mathbf{u}_{1}, \mathbf{u}_{2}\right)$ are created from a linear combination of the Bravais vectors $\left(\mathbf{a}_{1}, \mathbf{a}_{2}, \mathbf{a}_{3}\right)$. Here $\mathbf{u}_{1}=-\mathbf{a}_{1}+\mathbf{a}_{2}+\mathbf{a}_{3}$ and $\mathbf{u}_{2}=2 \mathbf{a}_{1}$. The two vectors are then projected from the three-dimensional (3D) representation to a $2 \mathrm{D}$ representation on a crystal surface. (b) Two cells of two different crystals are matched by applying the affine transformation $\mathbf{A}\left[\mathbf{u}_{1}, \mathbf{u}_{2}\right]=\left[\mathbf{v}_{1}, \mathbf{v}_{2}\right]$, where $\mathbf{A}=\mathbf{U P}$ consists of a rotation $\mathbf{U}$, and a strain matrix $\mathbf{P}$. 
the following system of linear equations,

$$
\left[\begin{array}{ll}
A_{11} & A_{12} \\
A_{21} & A_{22}
\end{array}\right]\left[\begin{array}{ll}
u_{1 x} & u_{2 x} \\
u_{1 y} & u_{2 y}
\end{array}\right]=\left[\begin{array}{ll}
v_{1 x} & v_{2 x} \\
v_{1 y} & v_{2 y}
\end{array}\right] .
$$

Any square matrix can be decomposed into the product of an orthonormal matrix $\mathbf{U}$, and a positive definite symmetric matrix P. This is known as a polar decomposition [20]. The symmetric matrix defines the 2D strain tensor $\epsilon$ for deforming one cell into the other,

$$
\mathbf{P}=\mathbf{I}+\boldsymbol{\epsilon}=\left[\begin{array}{lr}
1+\epsilon_{x x} & \epsilon_{x y} \\
\epsilon_{x y} & 1+\epsilon_{y y}
\end{array}\right] .
$$

The first vectors are rotated along the $x$ axis s.t. $u_{1 y}=$ $v_{1 y}=0$. This can be done without loss of generality and leads to

$$
\begin{gathered}
A_{11}=\frac{v_{1 x}}{u_{1 x}}, \\
A_{12}=\frac{v_{2 x}}{u_{2 y}}-\frac{v_{1 x} u_{2 x}}{u_{1 x} u_{2 y}}, \\
A_{21}=0, \\
A_{22}=\frac{v_{2 y}}{u_{2 y}} .
\end{gathered}
$$

We now make the polar decomposition of $\mathbf{A}$ s.t. $\mathbf{A}=\mathbf{U P}$, where $\mathbf{U}$ is a rotation matrix because of the chosen projection to $\mathbb{R}^{2}$,

$$
\begin{gathered}
\mathbf{U}=s\left[\begin{array}{cc}
A_{11}+A_{22} & A_{12} \\
-A_{12} & A_{11}+A_{22}
\end{array}\right] \\
=\left[\begin{array}{cc}
\cos (\phi) & -\sin (\phi) \\
\sin (\phi) & \cos (\phi)
\end{array}\right], \\
\mathbf{P}=\mathbf{U}^{T} \mathbf{A},
\end{gathered}
$$

where $s$ is a factor which makes the columns of $\mathbf{U}$ unit vectors. The $\mathbf{U}$ matrix defines the counterclockwise rotation of the $\left[\mathbf{u}_{1}, \mathbf{u}_{2}\right]$ cell onto the $\left[\mathbf{v}_{1}, \mathbf{v}_{2}\right]$ cell by the angle $\phi=\left|\phi_{a}-\phi_{b}\right| / 2$ as shown in Fig. 1(b). Using this method, Eqs. (7)-(12) thus yield the strain matrix of any given cell combination.

\section{A. Algorithm}

We now explain the algorithm behind extracting the strain matrix of all the possible matches between two crystals. The procedure is illustrated in the flow chart in Fig. 2.

\section{Create $(i, j, k)$ list from $\ell_{\max }$}

The first step is to create all the possible lattice vectors of each crystal up to a specified maximum length $\ell_{\max }$. The vectors are created as integer combinations of the Bravais vectors of the crystal, as illustrated in Fig. 1(a),

$$
\mathbf{u}=i \mathbf{a}_{1}+j \mathbf{a}_{2}+k \mathbf{a}_{3}, \quad|\mathbf{u}|<\ell_{\max } .
$$

This will create a list of $(i, j, k)$ values for each crystal.

\section{Create vector pair list}

The next step is to combine the created vectors such that a list of unique surface cells is created for each crystal. This
FOR EACH CRYSTAL

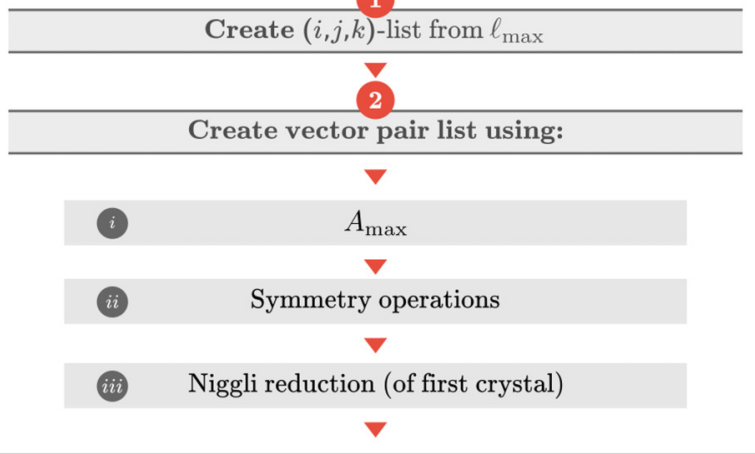

MATCHING THE CRYSTALS

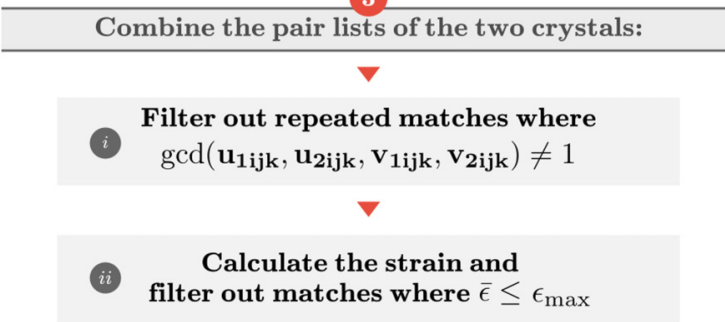

FIG. 2. Flow chart behind the algorithm for matching two crystals.

procedure uses two kinds of filters to remove equivalent surface cells from the list; the symmetry operations of the atom-free crystal [21] and Niggli reductions of the cells [22].

As a starting point, we create a list of symmetrically unique vectors of the crystal by applying the symmetry operations $\{\mathbf{S}\}$ to each vector in the generated $(i, j, k)$ list. Let $\mathbf{u}$ be the vector we apply the operations to and let $\{\mathbf{S u}\}$ be the set of all the vectors created when applying the symmetry operations. We define the canonical representation of this set as its least element with respect to the lexicographic ordering. When iterating through the $(i, j, k)$ list, noncanonical vectors are discarded.

To create the unique surface cells, we combine each vector from the original $(i, j, k)$ list with the vectors in the symmetry reduced $(i, j, k)$ list. The combination is discarded if the two vectors are parallel or if the area that they span exceeds $A_{\max }$. The symmetry operations of the crystal are then reapplied, this time on both vectors: $\left\{\mathbf{S}\left[\mathbf{u}_{1}, \mathbf{u}_{2}\right]\right\}$. Again, any noncanonical pairs are discarded.

The final test is to investigate whether the cell is a Niggli reduced cell. A Niggli reduced 2D cell fulfils

$$
\mathbf{u}_{1} \cdot \mathbf{u}_{1} \leqslant \mathbf{u}_{2} \cdot \mathbf{u}_{2}, \quad \mathbf{u}_{1} \cdot \mathbf{u}_{2} \leqslant \frac{1}{2} \mathbf{u}_{1} \cdot \mathbf{u}_{1} .
$$

The cells that are not Niggli reduced are discarded. This is only done for the first crystal, since a Niggli reduced cell may be strained into a non-Niggli reduced cell when the two crystals are combined. After these calculations, we have created a list of surface cells for each crystal and the next step is to combine these two lists. 


\section{Combine the pair lists of the two crystals}

When the cells of the two crystals are matched, the first step is to filter out repeated matches. As an example, let a $\left[\mathbf{u}_{1}, \mathbf{u}_{2}\right]$ cell of the first crystal be combined with a $\left[\mathbf{v}_{1}, \mathbf{v}_{2}\right]$ cell where $\mathbf{u}_{1 i j k}=(1,0,0), \mathbf{u}_{2 i j k}=(2,0,1), \mathbf{v}_{1 i j k}=(0,0,2)$, and $\mathbf{u}_{2 i j k}=(4,4,2)$. This combination is equivalent to the $((2,0,0),(4,0,2),(0,0,4),(8,8,4))$ combination. We avoid investigating both combinations by discarding any combinations whose greatest common divisor, $\operatorname{gcd}\left(\mathbf{u}_{1 i j k}, \mathbf{u}_{2 i j k}, \mathbf{v}_{1 i j k}, \mathbf{v}_{2 i j k}\right)$, is not 1 . After this preliminary test, the strain matrix of the match is calculated using the method explained in the beginning of this section.

We now define a measure for the average strain of a match,

$$
\bar{\epsilon}=\sqrt{\frac{\epsilon_{x x}^{2}+\epsilon_{y y}^{2}+\epsilon_{x x} \epsilon_{y y}+\epsilon_{x y}^{2}}{4}},
$$

where $\epsilon_{x x}, \epsilon_{x y}$, and $\epsilon_{y y}$ are the components of the 2D strain tensor shown in Eq. (6). This average strain is an invariant of the strain tensor, since $4 \bar{\epsilon}^{2}=\operatorname{Tr}(\boldsymbol{\epsilon})^{2}-\operatorname{det}(\boldsymbol{\epsilon})$. Matches with an average strain below a given strain threshold $\varepsilon_{\max }$ are kept.

This concludes the algorithm for finding all the matches between two crystals. The parameters determining which matches to include in the search are $\ell_{\max }, A_{\max }$, and $\varepsilon_{\max }$. These parameters help to filter out the cells that wouldn't create a physically meaningful interface. The area and length threshold ensures that we don't investigate unreasonably large or narrow cells and the strain threshold filters out the most strained matches.

The algorithm is implemented using $\mathrm{C}++$ with a Python interface and has good performance; finding all matches between a InAs fcc crystal and a Cobalt hep crystal with the parameters, $\ell_{\max }=50 \AA, A_{\max }=200 \AA^{2}$, and $\epsilon_{\max }=2 \%$, takes approximately $20 \mathrm{~min}$ on a normal laptop. The algorithm is available in VIRTUAL NANOLAB version ATK-VNL-2017 [23].

\section{MATCHING OF A FCC CRYSTAL WITH A BCC CRYSTAL}

In the previous section, we have explained how to match two specific crystals with fixed lattice parameters. Here, we discuss the matching of any fcc crystal with any bcc crystal. To this end, we introduce an isotropic scaling parameter $k$, which is applied to the Bravais vectors of the bcc crystal. This scaling parameter can then be defined as the ratio between the lattice constants of the two crystals, $k=a_{\mathrm{fcc}} / a_{\mathrm{bcc}}$. The effect of $k$ on the strain matrix is linear,

$$
\mathbf{A}=k \mathbf{U P}=\mathbf{U}\left[\begin{array}{cc}
k\left(1+\epsilon_{x x}\right) & k \epsilon_{x y} \\
k \epsilon_{x y} & k\left(1+\epsilon_{y y}\right)
\end{array}\right] .
$$

This means that the effect of the scaling parameter on the average strain can be described by the simple analytical relation,

$$
\begin{aligned}
4 \bar{\varepsilon}^{2}(k)= & k^{2}\left(\epsilon_{x x}^{2}+\epsilon_{y y}^{2}+\epsilon_{x x} \epsilon_{y y}+\epsilon_{x y}^{2}\right)+k^{2}\left(3 \epsilon_{x x}+3 \epsilon_{y y}+3\right) \\
& -k\left(3 \epsilon_{x x}+3 \epsilon_{y y}+6\right)+3,
\end{aligned}
$$

where the strain tensor components are referring to the case of $k=1$. This relation allows us to calculate the average strain of a match at any $k$ value once the strain matrix has been (a) [011] [113] [112] [023] [123] [013]

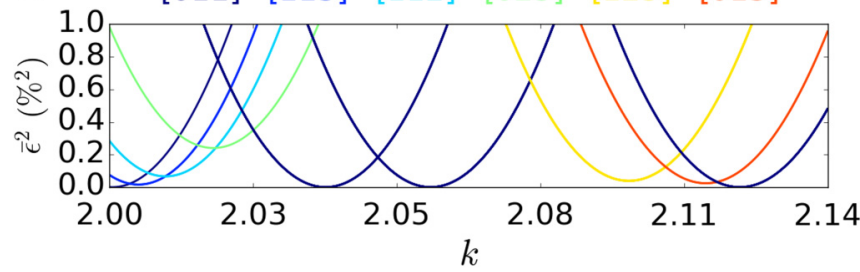

(b)

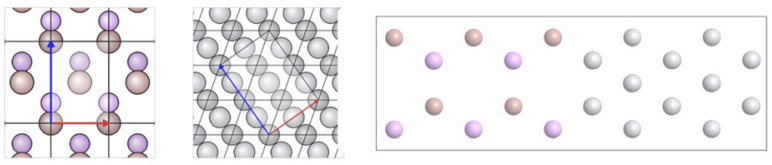

(c)

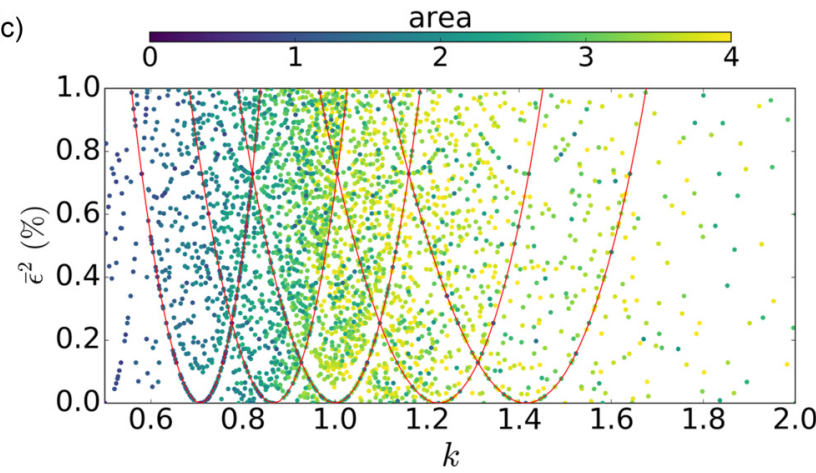

FIG. 3. The matches between a fcc and a bcc crystal. $k$ represents the scaling between the two lattice constants, $k=a_{\mathrm{fcc}} / a_{\mathrm{bcc}}$. (a) The $\bar{\varepsilon}^{2}(k)$ relation of (17) for optimal matches involving the [110] surface of the fcc crystal. The color of the curves represents different surfaces of the bcc crystal. (b) The surface cells and corresponding interface of the perfect $k=2$ match shown on (a). Illustrated here for InAs with vanadium. (c) Scatter plot of matches where the minima of the strain parabola gets below $10 \%$. The dots represent the minimal strain of the match and the $k$ value where this strain occurs. We have chosen $\ell_{\max }$ and $A_{\max }$ as four times the lattice constant of the unstrained crystal. The color represents the area of the fcc surface cell of the match.

calculated for any one specific value of $k$. Equation (17) defines a strain parabola; the minimal strain of a match, along with the corresponding $k$ value, can be found from the minimum of this parabola.

The strain parabolas for the optimal matches [24] between an fcc[110] surface and a bcc crystal with $k$ values between 2.0 and 2.14 are illustrated in Fig. 3(a). This range is of relevance for the matching of a InAsSb alloy with vanadium. It can be seen that the two [110] surfaces have perfect matches at $k=2.0,2.04,2.06$, and 2.12. The surface cells and corresponding interface of the perfect $k=2.0$ match are illustrated in Fig. 3(b) for InAs and vanadium. This $k$ value can be obtained, when matching, e.g., InAs $\mathrm{An}_{0.96} \mathrm{Sb}_{0.004}$ to vanadium or $\mathrm{Ga}_{0.786} \mathrm{In}_{0.214}$ As to iron. As such, this specific match reappears in the results of the next section where these two alloys are matched to a range of metals.

To obtain the full picture of the matches between a fcc and a bcc crystal, we use the algorithm described in Sec. III and apply the strain threshold to the minimal strain of a given match. It corresponds to the following flow chart in Fig. 2 but where step 3.ii. is altered such that after calculating the strain 
matrix, we use Eq. (17) to find the minimal strain of the match and only keep the matches where this is lower than the strain threshold. In this manner, we can retrieve the strain parabolas of all the relevant matches by doing a single calculation at $k=1$. We have chosen $\ell_{\max }$ and $A_{\max }$ as four times the lattice constant of the unstrained crystal and $\epsilon_{\max }=10 \%$.

Figure 3(c) shows the results of this general investigation. For each match, we plot the minimal strain and the corresponding $k$ value. Note, that the $y$ axis shows the squared strain, since this illustrates the general shape of the matches better. The structure shows curves where zero strain minimas appear, e.g., at the points $k=1 / \sqrt{2} \approx 0.71, k=\sqrt{3} / 2 \approx 0.87, k=1$, $k=\sqrt{3 / 2} \approx 1.22$, and $k=\sqrt{2} \approx 1.41$. The matches lying on these curves are related to a uniaxial strain between the two surface cells. The perfect matches at $k=1$ appear since this value corresponds to the [100] facets of the two cubic crystals fitting perfectly together. All other matches on this $k=1$ curve correspond to a match where one of the cells is rotated and then stretched in one direction only. The other zero strain points also represent some symmetry of the two crystals. The zero strain match at $k=1 / \sqrt{2}$, e.g., corresponds to matching the rotated bcc[100] surface with cell vectors $\mathbf{v}_{1}=\left[\sqrt{2} a_{\mathrm{bcc}}, 0\right]$ and $\mathbf{v}_{2}=\left[0, \sqrt{2} a_{\mathrm{bcc}}\right]$ to a [100] facet of the fcc crystal. The other matches lying on this curve represents a uniaxial strain on top of this perfect match where one of the cells is rotated, stretched in both directions by $\sqrt{2}$, and then stretched in one direction afterwards. The matches lying on a specific curve are thus related by having a fixed eigenvalue of the $\mathbf{P}$ matrix of $1 / k_{\mathrm{zsm}}$, where $k_{\mathrm{zsm}}$ is the $k$ value at the zero strain minimum of the curve. For example, all matches on the $k=1 / \sqrt{2}$ curve have $\mathbf{P}$ matrices with the eigenvalue $\sqrt{2}$.

Two things are important to note about this plot. First, the length and area limits of the vectors and cells determine the density of the found matches. Without these limits the entire $(\bar{\epsilon}, k)$ space would be filled with points. Secondly, the constraining to cubic structures lead to points with nonzero strain; if the crystal structures were allowed to vary arbitrarily, all points would have zero strain.

These results demonstrate that the method represents a general tool for crystal matching. The strength of the method is that it only relies on the geometry of the crystals. This makes it possible to calculate results for two arbitrary crystals and apply these results to all interfaces between materials of these crystal structures. Furthermore, it is an ideal tool for investigating alloy crystals where the lattice parameter can be varied as the composition of the alloy is changed. This will be the subject of the next section.

\section{INTERFACES BETWEEN SEMICONDUCTOR ALLOYS AND METALS}

We apply the method to two different semiconductor alloys, InAs ${ }_{1-x} \mathrm{Sb}_{x}$ and $\mathrm{Ga}_{x} \mathrm{In}_{1-x} \mathrm{As}$, and match their surfaces with those of 10 different metals (Al, Ni, $\mathrm{Cu}, \mathrm{Ag}, \mathrm{Au}, \mathrm{Pb}, \mathrm{V}, \mathrm{Fe}$, $\mathrm{Nb}$, and $\mathrm{Co}$ ). In particular, we study how the orientation and strain of the metallic surface depends on the lattice parameter of the semiconductor surface, which can be tuned by changing the mole fraction. The two alloys form a zincblende crystal

\begin{tabular}{|cccccccccccc|}
\hline & {$[013]$} & $\triangleleft$ & {$[133]$} & $\square$ & {$[113]$} & $\diamond$ & {$[233]$} & $\diamond$ & {$[010]$} & $\mathrm{I}$ & {$[332]$} \\
$\nabla$ & {$[011]$} & $\triangleright$ & {$[012]$} & $\diamond$ & {$[112]$} & 0 & {$[223]$} & + & {$[110]$} & - & {$[122]$} \\
$\Delta$ & {$[111]$} & $\bigcirc$ & {$[001]$} & $\star$ & {$[023]$} & $\diamond$ & {$[123]$} & $\times$ & {$[331]$} & & \\
\hline
\end{tabular}

(a)

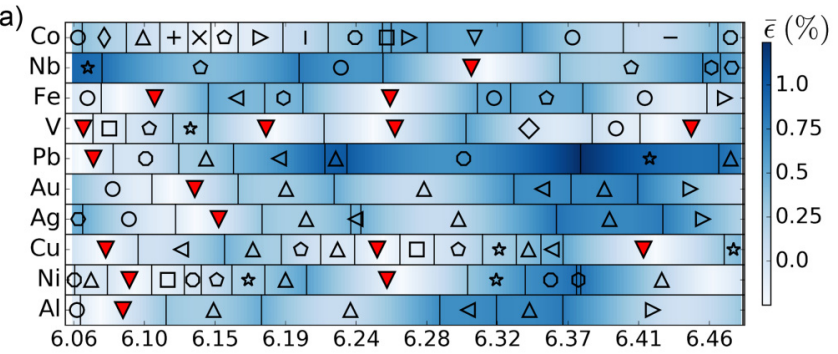

(b)

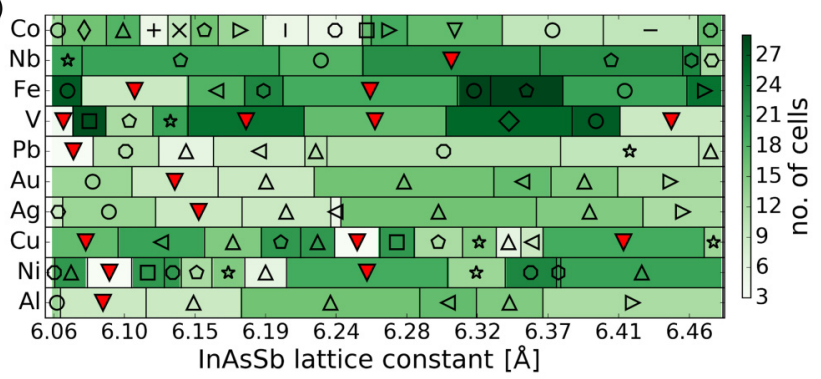

FIG. 4. (a) The InAsSb[1ํㅣㄹ surface matched with 10 different metals. Each block represents the match which results in the lowest average strain between the two materials. The background color shows the strain in percent and the markers show the involved metal surface. Filled markers denote a match where zero strain can be obtained. (b) Same as (a) but where the background color shows the area given by the number of alloy surface unit cells of the match.

and the relation between the lattice constant and mole fraction $x$ can be approximated by the linear relations [25]:

$$
\begin{aligned}
& a_{\mathrm{InAsSb}}(x)=6.0583+0.4207 x \\
& a_{\text {GaInAs }}(x)=6.0583-0.405 x
\end{aligned}
$$

We use the experimentally determined lattice constants [26] of the metals and strain the metal surfaces to match the alloy surfaces. The chosen parameters [27] are $\ell_{\max }=50 \AA, A_{\max }=$ $200 \AA^{2}$, and $\epsilon_{\max }=2 \%$. In addition, we set a limit on the Miller index of the crystal surfaces. If the highest value in the Miller index is above the threshold, $m_{\max }=3$, the match is discarded. The matches are calculated for a single value of the mole fraction and the scaling relation (17) of Sec. IV is then used to get the results for the rest of the $x$ values. This is possible, since each $x$ value directly corresponds to a $k$ value, $k=a_{\text {alloy }}(x) / a_{\text {metal }}$.

In Fig. 4, we show the matches involving the [1ํㅣㄹ surface of InAsSb. Each block in the plot corresponds to a certain match and a certain strain parabola in a plot like Fig. 3(a). The background of Fig. 4(a) therefore represents the strain value of the lowest lying strain parabola. Figure 3(a) shows InAsSb[1시 matched to vanadium and it is seen how the variation of the lattice parameter results in different optimal matches. For instance, from a perfect [110] match to a low-strain [113] match as the lattice constant is increased from 6.06 to 6.08 . 
(a)

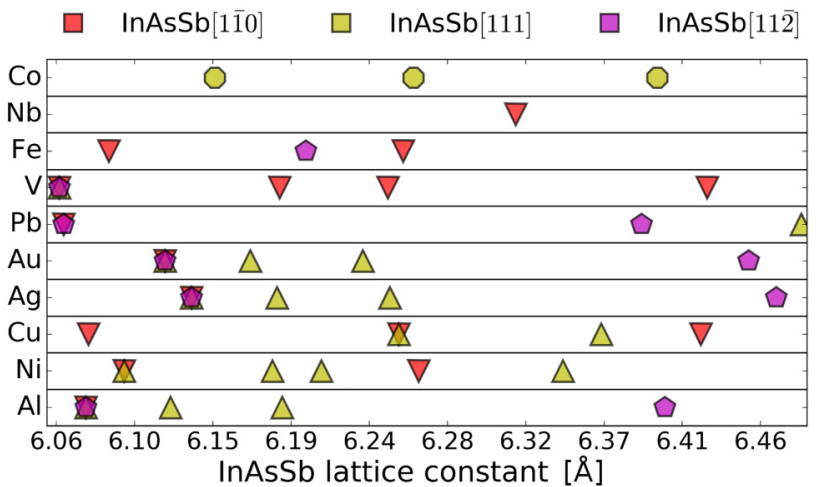

(b)

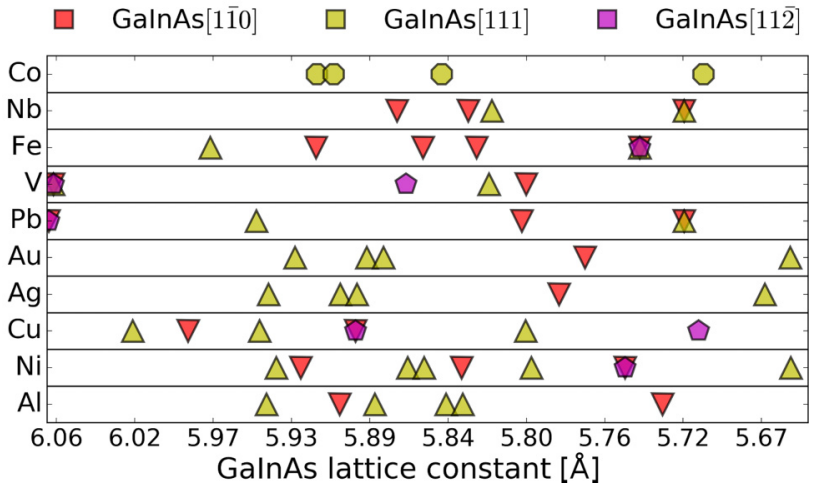

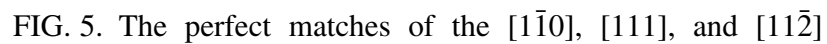
surfaces of (a) InAsSb and (b) GaInAs. The markers denote the metal surface (see Fig 4 for labels) and the color denotes the alloy surface. Each marker is placed at the alloy lattice constant which results in a zero strain match.

The area of the match, given as the number of alloy surface unit cells, is illustrated in Fig. 4(b). It is seen that several zero strain matches are possible between the InAsSb[1 10$]$ surface and those of the metals. Nickel, copper, lead, and vanadium even have perfect matches with very small unit cells, indicating that these interfaces will potentially be stable. The geometry of this small area match between InAsSb and vanadium can be seen in Fig. 3(b).

Figure 5 shows all the perfect matches of the [110], [111], and [112] surfaces of both InAsSb and GaInAs. The red triangles in Fig. 5(a) therefore represents the same matches as those highlighted in Fig. 4. The surfaces have been chosen since they typically terminate nanowires of the two investigated alloys. Many zero strain solutions are possible, especially for the [111] surfaces. Furthermore, it is seen that the perfect matches are distributed well across the range of lattice constants which should make it easier to realize some of these interfaces experimentally. Details of all the matches of the remaining two surfaces of InAsSb and the three surfaces of GaInAs is in the Supplemental Material [32].

The method has also been used to find matches between the two semiconductors and the superconductor $\mathrm{N} b_{1-x} \mathrm{~T} i_{x} \mathrm{~N}$. Since this material is an alloy in the $\mathrm{NaCl}$ structure, this is yet another case of varying the scaling parameter. We use a linear scaling of the NbTiN lattice constant in between the values
TABLE I. The perfect matches between NbTiN and the [110], [111], and [112̄] surfaces of InAsSb and GaInAs. The NbTiN surface is the same as the given alloy surface.

\begin{tabular}{llcccc}
\hline \hline InAsSb & $k$ value & No. of cells & GaInAs & $k$ value & No. of cells \\
\hline$[1 \overline{1} 0]$ & 1.414 & 2 & {$[1 \overline{1} 0]$} & 1.354 & 11 \\
{$[1 \overline{1} 0]$} & 1.5 & 9 & {$[111]$} & 1.271 & 21 \\
{$[111]$} & 1.363 & 13 & {$[111]$} & 1.309 & 12 \\
{$[111]$} & 1.453 & 19 & {$[111]$} & 1.323 & 14 \\
{$[111]$} & 1.5 & 9 & {$[111]$} & 1.333 & 16 \\
& & & {$[111]$} & 1.363 & 13 \\
& & & {$[11 \overline{2}]$} & 1.265 & 8 \\
& & & {$[11 \overline{2}]$} & 1.291 & 5 \\
& & & & 1.323 & 7 \\
\hline \hline
\end{tabular}

that can be found in the literature [28-31],

$$
a_{\mathrm{NbTiN}}(y)=4.30+0.17 y \quad y \in[0 ; 1] .
$$

With this definition, the $k$ value is given by $k=$ $a_{\text {alloy }}(x) / a_{\text {NbTiN }}(y)$. The used matching parameters are the same as for the investigation of the pure metals and the perfect match results can be seen in Table I. For InAsSb, we find two zero strain matches for the [1ㅣㅣㅇㅣ surface and the first of these matches also has a very small unit cell of only two alloy surface unit cells. For the [111] surface, we find three zero strain matches and the $k=1.5$ match has a reasonably small unit cell of nine surface cells. Plots like Figs. 4 and 5 showing all the matches between the semiconductors and NbTiN can be found in Supplemental Material [32].

\section{CONCLUSION}

We have presented a general method for matching two crystals at an interface. A scaling parameter between the lattice constants of two crystals was introduced. Using this scaling parameter, we can consider a general match between an fcc and a bcc crystal. Matches for any value of the lattice constant ratio can be found by performing a single calculation. The method was applied to the two semiconductor alloys InAs $\mathrm{A}_{1-x} \mathrm{Sb}_{x}$ and $\mathrm{Ga}_{x} \mathrm{In}_{1-x}$ As matched with a range of metals (Al, Ni, Cu, Ag, $\mathrm{Au}, \mathrm{Pb}, \mathrm{V}, \mathrm{Fe}, \mathrm{Nb}$, and $\mathrm{Co}$ ). The scaling parameter was used to tune the lattice constant of the alloys with their composition.

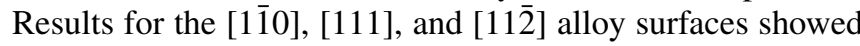
many perfect match solutions over a broad spectrum of the alloy lattice constant which is promising for realizing some of these matches experimentally. Finally, we have matched the two semiconductors to the superconducting alloy NbTiN. The results showed perfect matches with low surface cell areas for several of the investigated semiconductor surfaces.

\section{ACKNOWLEDGMENTS}

The authors acknowledges support from Innovation Fund Denmark under Project No. 5189-00082B "Atomicscale modeling of interfaces" and the Quantum Innovation Center (QUBIZ), Innovation Fund Denmark, and from the European Commissions Seventh Framework Programme (FP7/20072013), Grant Agreement IIIV-MOS Project 
No. 619326. We would like to thank Jesper Nygård and Thomas Sand Jespersen of the CQD at Copenhagen University for suggesting the idea of investigating semiconductor alloys, as well as Søren Smidstrup at QuantumWise A/S for the discussions and the help during the practical implementation of the matching method.
[1] A. Blom and K. Stokbro, J. Comput. Electron. 12, 623 (2013).

[2] V. Dusastre, J. Heber, F. Pulizzi, A. Stoddart, P. Pamies, and C. Martin, Nat. Mater. 11, 91 (2012).

[3] H. Kroemer, Rev. Mod. Phys. 73, 783 (2001).

[4] Y. Yang, K. Wang, H.-W. Liang, G.-Q. Liu, M. Feng, L. Xu, J.-W. Liu, J.-L. Wang, and S.-H. Yu, Sci. Adv. 1, e1500714 (2015).

[5] Y. Myung, D. M. Jang, T. K. Sung, Y. J. Sohn, G. B. Jung, Y. J. Cho, H. S. Kim, and J. Park, ACS Nano 4, 3789 (2010).

[6] P. K. Kasanaboina, S. K. Ojha, S. U. Sami, C. L. Reynolds, Jr., Y. Liu, and S. Iyer, Semicond. Sci. Technol. 30, 105036 (2015).

[7] A. L.-S. Chua, N. A. Benedek, L. Chen, M. W. Finnis, and A. P. Sutton, Nat. Mater. 9, 418 (2010).

[8] D. Stradi, L. Jelver, S. Smidstrup, and K. Stokbro, J. Phys.: Condens. Matter 29, 185901 (2017).

[9] P. Lazi, Comput. Phys. Commun. 197, 324 (2015).

[10] P. Krogstrup, N. L. B. Ziino, W. Chang, S. M. Albrecht, M. H. Madsen, E. Johnson, J. Nygård, C. M. Marcus, and T. S. Jespersen, Nat. Mater. 14, 400 (2015).

[11] W. Chang, S. M. Albrecht, T. S. Jespersen, F. Kuemmeth, P. Krogstrup, J. Nygård, and C. M. Marcus, Nat. Nanotechnol. 10, 232 (2015).

[12] M. T. Deng, S. Vaitieknas, E. B. Hansen, J. Danon, M. Leijnse, K. Flensberg, J. Nygård, P. Krogstrup, and C. M. Marcus, Science 354, 1557 (3016).

[13] A. P. Higginbotham, S. M. Albrecht, G. Kiranskas, W. Chang, F. Kuemmeth, P. Krogstrup, T. S. Jespersen, J. Nygård, K. Flensberg, and C. M. Marcus, Nat. Phys. 11, 1017 (2015).

[14] S. M. Albrecht, A. P. Higginbotham, M. Madsen, F. Kuemmeth, T. S. Jespersen, J. Nygård, P. Krogstrup, and C. M. Marcus, Nature (London) 531, 206 (2016).

[15] V. Mourik, K. Zuo, S. M. Frolov, S. R. Plissard, E. P. A. M. Bakkers, and L. P. Kouwenhoven, Science 336, 1003 (2012).

[16] H. Zhang, Ö Gül, S. Conesa-Boj, K. Zuo, V. Mourik, F. K. de Vries, J. van Veen, D. J. van Woerkom, M. P. Nowak, M. Wimmer, D. Car, S. Plissard, E. P. A. M. Bakkers, M. Quintero-Pérez, S. Goswami, K. Watanabe, T. Taniguchi, and L. P. Kouwenhoven, arXiv:1603.04069.

[17] D. Stradi, S. Barja, C. Díaz, M. Garnica, B. Borca, J. J. Hinarejos, D. Sánchez-Portal, M. Alcamí, A. Arnau, A. L. Vázquez de
Parga, R. Miranda, and F. Martín, Phys. Rev. B 88, 245401 (2013).

[18] M. Batzill, Surf. Sci. Rep. 67, 83 (2012).

[19] D. Stradi, S. Barja, C. Díaz, M. Garnica, B. Borca, J. J. Hinarejos, D. Sánchez-Portal, M. Alcamí, A. Arnau, A. L. Vázquez de Parga, R. Miranda, and F. Martín, Phys. Rev. Lett. 106, 186102 (2011).

[20] K. Shoemake and T. Duff, Proceedings of the Conference on Graphics Interface '92 (Morgan Kaufmann Publishers Inc., San Francisco, CA, 1992), pp. 258-264.

[21] We use the symmetry operations of the empty cell, since the algorithm does not take atomic positions into account.

[22] P. Niggli, Krystallographische und Strukturtheoretische Grundbegriffe (Akademische Verlagsgesellschaft, Leipzig, 1928).

[23] http://www.quantumwise.com; the VIRTUAL NANOLAB is freely available for academic researchers.

[24] The matching parameters $\ell_{\max }, A_{\max }, \varepsilon_{\max }$, and $m_{\max }$ are chosen as for the alloy investigations in Sec. V.

[25] I. P.-T. Institute, Electronic archive New semiconductor materials Characteristics and properties, http://www. ioffe.ru/SVA/NSM/Semicond/.

[26] C. Kittel, Introduction to Solid State Physics, 8th ed. (Wiley, New York, 2004).

[27] The area constraint is set for the cell size when using the lowest lattice constant of each alloy.

[28] K. Makise, H. Terai, M. Takeda, Y. Uzawa, and Z. Wang, IEEE Trans. Appl. Supercond. 21, 139 (2011).

[29] P. Bosland, F. Guemas, M. Juillard, M. Couach, and A. Khoder, in Proceedings of the Fifth Workshop on RF Superconductivity (DESY, Hamburg, 1991), p. 497.

[30] T. Shiino, S. Shiba, N. Sakai, T. Yamakura, L. Jiang, Y. Uzawa, H. Maezawa, and S. Yamamoto, Supercond. Sci. Technol. 23, 045004 (2010).

[31] H. Myoren, T. Shimizu, T. Iizuka, and S. Takada, IEEE Trans. Appl. Supercond. 11, 3828 (2001).

[32] See Supplemental Material at http://link.aps.org/supplemental/ 10.1103/PhysRevB.96.085306 for further results for interfaces between the semiconductor alloys InAsSb or GaInAs and metals or $\mathrm{NbTiN}$. 